UM-P-99/51, RCHEP-99/21

\title{
Geophysical constraints on mirror matter within the Earth
}

\author{
A.Yu.Ignatievt and R.R.Volkast \\ School of Physics, Research Centre for High Energy Physics, University of Melbourne, Victoria \\ 3010, Australia
}

\begin{abstract}
We have performed a detailed investigation of geophysical constraints on the possible admixture of mirror matter inside the Earth. On the basis of the Preliminary Reference Earth Model (PREM) - the "Standard Model" of the Earth's interior - we have developed a method which allows one to compute changes in various quantities characterising the Earth (mass, moment of inertia, normal mode frequencies etc.)due to the presence of mirror matter. As a result we have been able to obtain for the first time the direct upper bounds on the possible concentration of the mirror matter in the Earth. In terms of the ratio of the mirror mass to the Earth mass a conservative upper bound is $3.8 \times 10^{-3}$. We then analysed possible mechanisms (such as lunar and solar tidal forces, meteorite impacts and earthquakes) of exciting mirror matter oscillations around the Earth centre. Such oscillations could manifest themselves through global variations of the gravitational acceleration at the Earth's surface. We conclude that such variations are too small to be observed. Our results are valid for other types of hypothetical matter coupled to ordinary matter by gravitation only (e.g. the shadow matter of superstring theories).

95.35.+d, 91.35.-x, 11.30.Er, 12.60.-i, 14.80.-j
\end{abstract}

Typeset using REVTEX 


\section{INTRODUCTION}

Recently there has been considerable interest in the study of particle physics implications of the SuperKamiokande data on atmospheric neutrinos [1] as well as the results of other neutrino experiments [2]. The main purpose of such investigations has been to understand what theories could be responsible for the observed experimental features which give strong evidence for large angle neutrino oscillations. One of these theories is the Exact Parity Model (EPM) which introduces parity or "mirror" partners for all ordinary particles (except the graviton) and thus restores the parity invariance apparently broken by weak interactions [3 [7]. The Exact Parity Model predicts pairwise maximal mixing between ordinary and mirror neutrinos and provides a basis for interpretation of atmospheric neutrino and solar neutrino data [5].

An important question that arises naturally is whether or not the existence of mirror particles can lead to other observable consequences. In this work our objective is to find constraints on the possible concentration of mirror particles in the Earth. These constraints are a necessary step in the search for mirror world manifestations in terrestrial experiments. In particular, the presence of mirror matter in the Earth could lead to the regeneration of ordinary neutrinos from mirror neutrinos and consequently to the suppression of the day-night effect in the neutrino data.

Two main approaches to our problem are possible. First, one can trace the fate of the mirror particles starting from the early Universe epoch through the structure formation periods (galaxies, solar system and finally the Earth). Second, we can use geophysical data to get a more direct limit on the concentration of mirror matter in the Earth regardless of possible cosmological bounds.

It has been suggested that considerations based on the structure formation theory disfavour a significant presence of mirror matter in the Earth (see Blinnikov and Khlopov in [6]; Kolb, Seckel and Turner in [6]). However, as our knowledge of the structure formation is still incomplete, it is important to develop a geophysical approach as an independent, complementary tool of analysis exploiting the wide and rich variety of observational data accumulated in the Earth sciences.

This approach will be applicable not only to the specific EPM model, but also to any other theory predicting the existence of a new world of particles which couples to the ordinary matter only through gravitational interaction. An example is the shadow matter characteristic of superstring theories.

The plan of the paper is as follows. Section 2 summarises the main aspects of the "Standard Earth Model" called "Preliminary Reference Earth Model" (PREM). In Section 3 we analyse the possible effects of mirror matter within the context of PREM. Section 4 is devoted to the study of dynamical manifestations of mirror matter and comparison with gravimetric data. Finally, we present our conclusions in Section 5.

\section{PRELIMINARY REFERENCE EARTH MODEL (PREM)}

The Preliminary Reference Earth Model (PREM) [8] is a mechanical model of the average internal structure of the Earth based, mainly, on the analysis of seismological data. It gives 
the radial distributions of mechanical properties (such as density, elastic moduli, pressure, gravity and others) in the Earth's interior.

The set of initial data used for constraining the model includes:

1. astronomic-geodetic data (radius, mass and moment of inertia of the Earth);

2. data on free oscillations and long-period surface waves (over 1000 eigenfrequencies are known);

3. body waves data ( $\sim 10^{6}$ arrival times have been registered).

Let us summarise the main analytical relations used in the construction of the model (for more details see e.g. [9, [10]). The velocities of the elastic waves are given by

$$
v_{p}(r)=\sqrt{\frac{K(r)+\frac{4}{3} \mu(r)}{\rho(r)}}, \quad v_{s}(r)=\sqrt{\frac{\mu(r)}{\rho(r)}},
$$

where $v_{p}$ is the velocity of longitudinal waves, $v_{s}$ is the velocity of transverse waves, $K$ is the bulk modulus (or incompressibility) and $\mu$ is the shear modulus. These velocities can be found from seismological observations as functions of radius $r$. Measurements of wave velocities supply the ratios $K / \rho$ and $\mu / \rho$. To obtain $\rho$ independently, the Adams-Williamson equation,

$$
-\frac{d \rho}{d r}=\frac{G \rho(r)}{r^{2}\left(v_{p}^{2}(r)-\frac{4}{3} v_{s}^{2}(r)\right)} \int_{0}^{r} 4 \pi a^{2} \rho(a) d a,
$$

must be used. This equation expresses the condition of mechanical equilibrium between the gravitational attraction and the pressure due to elastic compression. G is Newton's constant, and the combination of the squared sound speeds in the denominator is called the seismic parameter $\Phi$ :

$$
\Phi(r) \equiv v_{p}^{2}(r)-\frac{4}{3} v_{s}^{2}(r)=\frac{K(r)}{\rho(r)}
$$

Equation (2) is valid for a chemically homogeneous layer with adiabatic temperature gradient.

Further, the connections between the density profile and the profiles of pressure and gravity are given by

$$
\frac{d P}{d r}=\Phi(r) \frac{d \rho}{d r}
$$

and

$$
g(r)=\frac{G}{r^{2}} \int_{0}^{r} 4 \pi a^{2} \rho(a) d a
$$

\section{STATIC CONSTRAINTS}

In this section, we will compute constraints on stationary mirror matter from PREM. A later section will consider dynamic manifestations of mirror matter. 


\section{A. Pedagogical warm-up exercise}

In order to get a feeling for what would go wrong if a substantial amount of mirror matter were present in the Earth, we consider a simple but unrealistic scenario first. Suppose the Earth is actually two concentric spheres, one ordinary and one mirror. Suppose also, for simplicity, that the mass density ratio $\rho_{1}(r) / \rho_{0}(r)$ of mirror to ordinary matter is independent of radius. This is clearly unrealistic and we will relax this assumption in the next subsection.

In this case, alterations due to mirror matter of the PREM equations (11, 2, 3, 田, 5) can be accomodated either by rescaling Newton's constant,

$$
G \rightarrow G^{\prime}=G\left(1+\frac{\rho_{1}}{\rho_{0}}\right)
$$

(throughout the paper quantities with index 0 will refer to ordinary matter while those with index 1 to mirror matter) or by keeping $G$ fixed and rescaling all of the other quantities used in PREM.

While the first procedure (rescaling $G$ ) seems to be the simplest, the second procedure reveals the physical modifications required in the presence of mirror matter more clearly and allows one to use PREM results directly.

From the formulas (1) and the Adams-Williamson equation (2) we deduce that the effective parameters can be defined as

$$
\begin{gathered}
\rho_{\text {eff }}=\rho_{0}+\rho_{1}, \\
P_{e f f}=P_{0}\left(1+\frac{\rho_{1}}{\rho_{0}}\right), \\
\mu_{e f f}=\mu_{0}\left(1+\frac{\rho_{1}}{\rho_{0}}\right), \\
K_{e f f}=K_{0}\left(1+\frac{\rho_{1}}{\rho_{0}}\right), \\
\Phi_{e f f}=\frac{G}{r^{2}} \int_{0}^{r} \frac{4 \pi a^{2} \rho_{e f f}(a) d a,}{v_{p, e f f}^{2}-\frac{4}{3} v_{s, e f f}^{2}=\frac{K_{e f f}}{\rho_{e f f}}=\frac{K_{0}}{\rho_{0}}=v_{p, 0}^{2}-\frac{4}{3}} v_{s, 0}^{2} \equiv \Phi_{0}, \\
v_{s, e f f}=v_{s, 0}=\sqrt{\frac{\mu_{e f f}(r)}{\rho_{e f f}(r)}} . \\
v_{p, e f f}=v_{p, 0}=\sqrt{K_{e f f}(r)+\frac{4}{3} \mu_{e f f}(r)}
\end{gathered}
$$


With these definitions the form of the Adams-Williamson equation does not change:

$$
-\frac{d \rho_{e f f}}{d r}=\frac{\rho_{e f f} g_{e f f}}{\Phi_{e f f}}
$$

Also, the usual equation for the bulk modulus holds true in terms of the effective quantities:

$$
\frac{d \rho_{e f f}}{d P_{e f f}}=\frac{\rho_{e f f}}{K_{e f f}} .
$$

This demonstrates self-consistency of the rescaling procedure.

Now, for illustrative purposes, consider the case of a 50\%-50\% mixture of ordinary and mirror matter. The effective values for the density, incompressibility and pressure at the Earth centre can be taken directly from PREM data:

$$
\rho_{\text {eff }}=13.1 \mathrm{~g} / \mathrm{cm}^{3}, \quad K_{\text {eff }}=14.2 \mathrm{Mb}, \quad P_{\text {eff }}=3.64 \mathrm{Mb} .
$$

Correspondingly, the values for the ordinary matter (at the centre) are obtained by a factor of 2 rescaling:

$$
\rho=6.55 \mathrm{~g} / \mathrm{cm}^{3}, \quad K=7.1 \mathrm{Mb}, \quad P=1.82 \mathrm{Mb} .
$$

With these values, iron is definitely ruled out as the main component of the core and, therefore, the Earth as a whole. However, from independent evidence we know that iron is one the most abundant elements in the Earth. In addition, no other element (with significant abundance) can have the properties required by Eq. (18). For instance, silicon (at zero pressure) has a lower density than iron, but its incompessibility is greater than that of iron. Therefore the 50\%-50\% mixture of ordinary and mirror matter in the Earth is incompatible with the observational data encoded by the PREM model taking into account knowledge of terrestrial chemistry.

\section{B. Modified Adams-Williamson equation}

Consider now the realistic case where the mirror matter density does not follow the

ordinary density. Then the density of the ordinary matter (indexed by 0) would obey the modified Adams-Williamson equation:

$$
-\frac{d \rho_{0}}{d r}=\frac{G \rho_{0}}{r^{2}\left(v_{p 0}^{2}-\frac{4}{3} v_{s 0}^{2}\right)} \int_{0}^{r} 4 \pi a^{2}\left(\rho_{0}(a)+\rho_{1}(a)\right) d a .
$$

Also, we have to require that the total mass of the Earth and the moment of inertia are equal to their observed values:

$$
\begin{gathered}
\int_{0}^{R_{\oplus}} 4 \pi a^{2}\left(\rho_{0}(a)+\rho_{1}(a)\right) d a=M_{\oplus}, \\
\frac{8 \pi}{3 M_{\oplus} R_{\oplus}^{2}} \int_{0}^{R_{\oplus}} a^{4}\left(\rho_{0}(a)+\rho_{1}(a)\right) d a=I,
\end{gathered}
$$


where

$$
M_{\oplus}=5.974 \times 10^{24} \mathrm{~kg}, \quad I=0.3308 .
$$

Let us start with the simplest case: assume that the mirror matter forms a ball of uniform density $\rho_{1}$ with a radius equal to the radius of the inner core $\left(R_{\text {inner core }}=1221 \mathrm{~km} \approx R_{\oplus} / 5\right)$. Within PREM the density profile in the inner core is given by the following function:

$$
\rho_{P R E M}(r)=\rho_{P R E M}(0)-q \frac{r^{2}}{R_{\oplus}^{2}},
$$

where

$$
\rho_{P R E M}(0)=13.09 \mathrm{~g} / \mathrm{cm}^{3}, \quad q=8.84 \mathrm{~g} / \mathrm{cm}^{3} .
$$

Therefore, we look for the solution of the modified Adams-Williamson equation in the following form

$$
\rho_{0}(r)=\rho_{P R E M}(0)-\epsilon-(q+\delta) \frac{r^{2}}{R_{\oplus}^{2}},
$$

assuming that the mirror density $\rho_{1}$ is small compared to the Earth central density in PREM model $\rho_{P R E M}(0)$. Solving the system of equations $(19$, 25) we find:

$$
\epsilon \approx 0.84 \rho_{1}, \quad \delta \approx 0.9 \rho_{1}
$$

Thus we see that if we wish to add to the Earth some amount of mirror matter then consistency with the equilibrium equation requires that the density of ordinary matter be decreased (as compared with PREM density) by approximately the same amount. Also, it can be shown that if Eq. (26) holds then the constraints due to the Earth mass and the moment of inertia are automatically satisfied as long as $\rho_{1}<0.77 \mathrm{~g} / \mathrm{cm}^{3}$.

Such a decrease of ordinary matter density can be achieved in one of two ways:

1) by changing the chemical composition of the core so that the new composition has lower density than the standard;

2) by decreasing the pressure so that the density of the standard core composition is lowered.

Before we consider these two possibilities let us review briefly the subject of the standard core composition (see e.g. [10]). Information about the chemical composition of the core is obtained by comparing the mechanical characteristics given by the PREM model with the properties of various substances under high pressure. In this way it has been established that the core characteristics are close but not equal to those of iron. There is sufficient evidence to conclude that some lighter element should be added to iron in order to satisfy the geophysical constraint on the core composition. Sulfur and oxygen appear to be the strongest candidates for that role although other elements (such as carbon, nitrogen etc) cannot be ruled out at present. As examples, cores containing 6-12\% sulfur or 7-8\% oxygen (by mass) have been proposed as possible compositions consistent with the PREM model.

In order to accomodate mirror matter according to the first method above, we should increase the admixture of light elements as compared with the standard levels. However, 
such an increase would typically raise the sound velocity in the core (for more details see [10]). Requiring that the sound velocity is equal within accuracy of about $0.3 \%$ to the observed value (see [8]) we can estimate that

$$
\epsilon \lesssim 0.18 \mathrm{~g} / \mathrm{cm}^{3} .
$$

Next, in the second method we have to lower the pressure in order to obtain lower density of the (ordinary) matter. Lowering the pressure would lead to a decrease of the sound velocity and from the same requirement as above we can conclude that

$$
\epsilon \lesssim 0.06 \mathrm{~g} / \mathrm{cm}^{3} \text {. }
$$

Then, using Eq. (26, 27, 28) we can obtain the upper bound on the mirror density in the inner core

$$
\rho_{1} \lesssim \frac{1}{0.84} \max \{0.18,0.06\} \mathrm{g} / \mathrm{cm}^{3}=0.21 \mathrm{~g} / \mathrm{cm}^{3} .
$$

Translated to the upper limit on the ratio of the mirror mass to the total mass of the Earth, this becomes:

$$
\frac{M_{1}}{M_{\oplus}} \lesssim 2.7 \times 10^{-4}
$$

\section{Constraints from free oscillations of the Earth}

Let us consider now the effect of changing the ordinary density on the Earth eigenfrequencies. Using Eq.(A2-A6) of Ref. [8] and Eq.(41) of Ref. [11] it can be shown that the relative change of period of the spheroidal ${ }_{0} S_{0}$ mode as a result of changing the inner core density by $\epsilon$ would be

$$
\frac{\delta T}{T} \approx-\frac{0.026 \epsilon}{\rho_{P R E M}(0)} .
$$

Requiring that this shift of period be less than the fitting accuracy, $0.05 \%$, we obtain a bound on the allowed change of the ordinary matter density in the inner core:

$$
\frac{\epsilon}{\rho_{P R E M}(0)} \lesssim 1.9 \times 10^{-2}, \quad \epsilon \lesssim 0.26 \mathrm{~g} / \mathrm{cm}^{3} .
$$

Using Eq. (26) this bound can be translated into a limit on the mirror matter density in the inner core:

$$
\frac{\rho_{1}}{\rho_{P R E M}(0)} \lesssim 2.3 \times 10^{-2}, \quad \rho_{1} \lesssim 0.3 \mathrm{~g} / \mathrm{cm}^{3} .
$$

In terms of the total mass of the mirror matter $M_{1}$ we can rewrite Eq. (33) as

$$
\frac{M_{1}}{M_{\oplus}} \lesssim 3.8 \times 10^{-4} \text {. }
$$

Comparing Eqs. (30) and (34) we see that the difference between these upper limits is not very significant, although Eq. (30) is perhaps a less reliable estimate than Eq. (34) because of the incomplete knowledge of mechanical properties of various materials at high pressures characteristic for the Earth's centre. For these reasons we interpret Eq. (34) as our final conservative upper bound on the mirror matter mass located inside the inner core of the Earth. 


\section{Arbitrary radius of the mirror matter ball}

So far we have considered the case when mirror matter is contained completely inside the inner core of the Earth. To justify such an assumption one would need to know the detailed macroscopic properties of mirror matter (such as equation of state, chemical composition etc.); then using the condition of mirror matter equilibrium one could obtain the relation between the mirror matter and its radius. If we do not want to rely on such additional information than we have to regard the radius of the mirror ball $R_{1}$ as a free parameter of our model. Of course the resulting constraints will be weaker than they could be otherwise.

For simplicity we will restrict ourselves to the two characteristic values of $R_{1}$ in addition to the case $R_{1}=R_{\text {inner core }}$ considered before: first, $R_{1}=R_{\text {outer core }} \approx 0.55 R_{\oplus}$, and, second, $R_{1}=R_{\text {lower mantle }} \approx 0.89 R_{\oplus}$. The second choice is motivated by the fact that for radii larger than $R_{\text {lower mantle }}$ the Adams-Williamson equation is not valid anymore.

Also, we will assume that the mirror matter has a uniform density $\rho_{1}$ while the density of the ordinary matter differs from its PREM value by a radius-independent correction $\delta \rho_{0}$ for

$r \leq R_{1}$ and coincides with the PREM value for $r>R_{1}$. Although both these assumptions are clearly unrealistic, they nevertheless give us a self-consistent approximation scheme because both $\rho_{1}$ and $\delta \rho_{0}$ are small and therefore the effect of their radial dependence would be a second-order correction.

The precise details here depend on the chemical composition, the equation of state and other thermodynamic parameters for mirror matter. For instance, if we are given the equation of state for the mirror matter then (using some additional simplifying assumptions, such as chemical homogeneity, neglecting temperature etc.) we could find the density profile of the mirror matter (in particular, its central density) from the equation of mechanical equilibrium. However, our goal in this paper was to obtain limits on the mirror matter that would be independent of such particularities.

In any case, our method allows one to calculate the upper limit on the mirror matter mass for an arbitrary distribution of mirror matter $\rho_{1}(r)$.

As before, our constraints on the mass of the mirror matter will be based on 4 pieces of information:

1)mass of the Earth and its coefficient of inertia;

2)validity of the modified Adams-Williamson equation;

3)periods of Earth's free oscillations;

4) velocities of elastic waves.

Generally speaking, points 1) and 2) tell us that the correction to the ordinary matter density should be approximately equal to the density of the mirror matter $\delta \rho_{0} \simeq \rho_{1}$. Next, using that equality we can compute the shift of the period for the ${ }_{0} S_{0}$ mode and then obtain the upper limit on the mirror mass. From what follows it will be evident that we do not need to find an exact relation between $\rho_{1}$ and $\delta \rho_{0}$ as it would not significantly change the final constraints. Therefore, the validity of the modified Adams-Williamson equation (MAWE for short) can be analysed in a simpler manner than that of Sec. B.

As a criterion of validity of MAWE we can require that the actual mass of the Earth (that is, the ordinary mass plus the mirror mass) inside any radius $r \leq R_{1}$ should be equal, with the accuracy of $1 \%$ [8], to the PREM mass of the Earth (within the same radius): 


$$
w \equiv\left|\frac{M(r)}{M(r)_{P R E M}}-1\right| \lesssim 1 \%
$$

where

$$
M(r)=M(r)_{P R E M}+\int_{0}^{r} 4 \pi a^{2}\left(\rho_{1}-\delta \rho_{0}\right) d a \simeq M(r)_{P R E M}+\frac{4}{3} \pi r^{3}\left(\rho_{1}-\delta \rho_{0}\right) .
$$

It is convenient to introduce the dimensionless ratio

$$
f=\frac{\rho_{1}-\delta \rho_{0}}{\bar{\rho}_{\oplus}}
$$

where $\bar{\rho}_{\oplus}=5.5 \mathrm{~g} / \mathrm{cm}^{3}$ is the average density of the Earth. In terms of this quantity the condition (35) can be rewritten as

$$
w=\left(\frac{r}{R_{\oplus}}\right)^{3}\left(\frac{M_{\oplus}}{M(r)_{P R E M}}\right) \times f \lesssim 0.01,
$$

for $r \leq R_{1}$.

Next, we require that the total mass of the Earth equal the observed value with an accuracy of $1.3 \times 10^{-4}[9]$ which translates into

$$
\left(\frac{R_{1}}{R_{\oplus}}\right)^{3} \times f \lesssim 1.3 \times 10^{-4}
$$

Further, we have to demand that the coefficient of inertia of the Earth equals its PREM value $I_{P R E M}=0.3308$ with an accuracy of $\Delta I / I \lesssim 3 \times 10^{-4}$. In terms of $f$ this condition reads

$$
\left(\frac{R_{1}}{R_{\oplus}}\right)^{3}\left(1-1.5\left(\frac{R_{1}}{R_{\oplus}}\right)^{2}\right) \times f \lesssim 3 \times 10^{-4} .
$$

We observe that this condition does not give us an independent constraint because it is satisfied automatically as long as inequality (39) is fulfilled.

Finally, we compute the shift of the period for the ${ }_{0} S_{0}$ normal mode of the Earth due to non-zero $\delta \rho_{0}$ using the same method as in Section C. In the case $R_{1}=R_{\text {outer core }}$ we find:

$$
\frac{\delta T}{T} \approx-0.136\left(\frac{\rho_{1}}{\bar{\rho}_{\oplus}}-f\right) .
$$

From inequality (39) we conclude that

$$
f<8 \times 10^{-4}
$$

With these values of $f$ the criterion (38) of MAWE validity is clearly fulfilled for all radii $r \leq R_{1}$. Next, requiring that the shift of period be less than the fitting accuracy $(0.05 \%)$, we obtain the upper limit on the mirror matter density

$$
\rho_{1} \lesssim 0.025 \mathrm{~g} / \mathrm{cm}^{3} .
$$


This translates into the following bound on the mirror matter mass:

$$
\frac{M_{1}}{M_{\oplus}} \lesssim 7.4 \times 10^{-4} \quad\left(\text { for } R_{1}=0.55 R_{\oplus}\right)
$$

Following the same procedure, in the case $R_{1}=R_{\text {lower mantle }}$ we obtain:

$$
\frac{\delta T}{T} \approx-0.096\left(\frac{\rho_{1}}{\bar{\rho}_{\oplus}}-f\right) .
$$

From (39) we find an upper bound on $f$ :

$$
f<1.8 \times 10^{-4}
$$

Again, the condition of MAWE validity, Eq. (38), is satisfied automatically with these $f$. Further, the upper limit on the mirror matter density $\rho_{1}$ becomes

$$
\rho_{1} \lesssim 0.03 \mathrm{~g} / \mathrm{cm}^{3}
$$

Correspondingly, the bound on the mirror matter is

$$
\frac{M_{1}}{M_{\oplus}} \lesssim 3.8 \times 10^{-3} \quad\left(\text { for } R_{1}=0.89 R_{\oplus}\right)
$$

Comparing our bounds (43) and (47) with the limit (29) we see that the latter limit is significantly weaker than the former two (although the limit (29) has been obtained for the inner core, it is also valid for the outer core because of the similarity of their chemical composition; we also would not expect substantial changes of this limit in the case of the lower mantle). Therefore we conclude that Eq. (34), Eq. (44), and Eq. (48) represent our final upper bounds on the mirror matter mass. Being the largest of the three, Eq. (48) can also be considered as the most conservative, radius-independent upper bound on the mirror matter mass in the Earth.

\section{DYNAMICAL MANIFESTATIONS OF MIRROR MATTER}

In this section we are going to analyze constraints on mirror matter that follow from the precision measurements of the Earth's gravitational field. Such constraints may arise if the mirror matter, for some reason, shifts away from the centre of the Earth.

The motion of the mirror matter in the Earth would be controlled mainly by the Earth's gravity field; inside the core, in a first approximation this field grows linearly with the radius:

$$
g \approx k r, \quad k=3.6 \times 10^{-6} s^{-2} .
$$

(The net tidal force exerted on the mirror matter by the Moon and the Sun is negligibly

small as will be discussed later.) Consequently, the period of the mirror matter motion inside the Earth $T_{1}$ would be independent of radius and given by

$$
T_{1}=\frac{2 \pi}{\sqrt{k}}=3311 \mathrm{~s} \approx 55 \min .
$$


This period gives us the time scale for the variation $\delta g$ of gravitational acceleration at the Earth's surface caused by the possible mirror matter motion.

To determine the amplitude of the gravity variation suppose that the amplitude of the mirror matter motion is $h$. Then the amplitude of the gravity variation is given by

$$
\frac{\delta g}{g} \simeq \frac{M_{1}}{M_{\oplus}} \frac{h}{R_{\oplus}} .
$$

The above equation holds exactly only for the circle on the Earth's surface which lies in the plane of the mirror matter motion. For points outside this circle Eq.(51) can still be used for order-of-magnitude estimates. Requiring that the gravity variation should not exceed the observational limit, $\delta g / g \lesssim 10^{-9}$ (see e.g. [12]) we obtain an upper bound on the amplitude of mirror matter motion:

$$
h \lesssim 1.7 \times\left(\frac{3.8 \times 10^{-3} M_{\oplus}}{M_{1}}\right) m .
$$

What physical factors could lead to the off-centre shift of the mirror matter? Let us start by discussing the possible effect of Moon's gravity. The two key quantities to be considered are the tidal torque and the net tidal force exerted on the mirror matter by the Moon. The effect of the tidal torque would be to slow down the spinning of the mirror matter, in analogy with the ordinary tidal torque that brakes the Earth's rotation. The details of the effect depend on the poorly known characteristics of the mirror matter such as its angular velocity, elastic and dissipative properties etc.; we will not dwell on these.

On the other hand, the net tidal force and the corresponding off-centre shift can be estimated without the knowledge of mirror matter properties. First of all we note that if the Earth was spherically symmetric then there would be no net tidal force acting on the mirror "ball" placed in the Earth's centre. However, if the oblateness of the Earth is taken into account then the net force at the centre is non-zero and thus the centre of the Earth is not an equilibrium position anymore. The new equilibrium position for the mirror matter can be found from the condition of balance between the net tidal force and the gravitational attraction of the mirror matter by the Earth.

To find the new equilibrium position it is convenient first to find the point in the Earth where the lunar tidal force vanishes ("the tidal centre"). Due to oblateness of the Earth the positions of the Earth's centre of mass and the tidal centre are shifted relative to each other by a short distance $b$ (hereafter we ignore the $18^{\circ}$ inclination of the Moon's orbit relative to the Earth's equatorial plane):

$$
b=J_{2} \frac{3 R_{\oplus}^{2}}{2 R} \approx 171 \mathrm{~m},
$$

where

$$
J_{2}=\frac{C-B}{M_{\oplus} R_{\oplus}^{2}} \simeq 1.08 \times 10^{-3}
$$

is the Earth's dynamical oblateness, $R \approx 3.84 \times 10^{8} \mathrm{~m}$ is the distance between the Moon and the Earth, $C$ and $B$ are the moments of inertia of the Earth with respect to the principal 
axes. Therefore the centre of mass of the mirror matter will move away from the Earth centre (and also away from the tidal centre) by the distance

$$
h \approx \frac{2 G m b}{k R^{3}}
$$

where $m$ is the Moon's mass. Inserting Eq.(53) into Eq.(55) we obtain

$$
h \approx \frac{3 G m J_{2} R_{\oplus}^{2}}{k R^{4}} \approx 8.1 \times 10^{-6} \mathrm{~m} .
$$

The off-centre shift of mirror matter would create periodic variations of the gravity acceleration on the surface of Earth:

$$
\frac{\delta g}{g} \simeq \frac{h}{R_{\oplus}} \frac{M_{1}}{M_{\oplus}} \approx 1.3 \times 10^{-12} \frac{M_{1}}{M_{\oplus}}
$$

which is far beyond the observational limits. Thus we have shown that the effect of a net tidal force due to the Moon is negligible. A similar result holds for the solar effect:

$$
\tilde{h} \approx \frac{3 G M_{\odot} J_{2} R_{\oplus}^{2}}{k \tilde{R}^{4}} \approx 10^{-8} \mathrm{~m}
$$

where $M_{\odot} \approx 2 \times 10^{30} \mathrm{~kg}$ is the solar mass, $\tilde{R} \approx 1.5 \times 10^{11} \mathrm{~m}$ is the distance between the Sun and the Earth. As expected, the effect of the net tidal force due to the Sun is even smaller than the lunar effect.

We now consider non-gravitational interactions that could possibly cause an off-centre shift of the mirror matter. Let us start by analysing the possible role of meteorites and meteor showers colliding with the Earth. Suppose that as a result of such a collision a momentum $p$ is transferred to the Earth. Then the mirror matter (assumed to be at rest in the centre) would receive an initial velocity $u=p / M_{\oplus}$ relative to the Earth. Therefore, the off- centre displacement would be equal to

$$
h_{c o l}=\frac{u}{\sqrt{k}}=\frac{p}{M_{\oplus} \sqrt{k}} .
$$

What could be the magnitude of $p$ ? The maximal velocity of a Sun-bound colliding object, relative to the Earth, is $v_{\max } \simeq 73 \mathrm{~km} / \mathrm{s}$. The heaviest meteorite found on the Earth has the mass $m_{\max } \simeq 60$ ton. Inserting these values into Eq. (59) we obtain:

$$
h_{c o l} \lesssim \frac{m_{\max } v_{\max }}{M_{\oplus} \sqrt{k}} \approx 3.8 \times 10^{-13} \mathrm{~m}
$$

The variations of surface gravity acceleration caused by such displacements are many orders of magnitude beyond observational accuracy. Note that the impact of meteor showers would be much less than the estimate (60) since the total mass of even the most copious showers is significantly less than $m_{\max }$.

In the case of still heavier meteorites which disperse after hitting the Earth the mass can be estimated only indirectly (see e.g. 13 ). For instance, the meteorite that created the Arizona crater (with diameter of $1207 \mathrm{~m}$ and depth $174 \mathrm{~m}$ ) had the estimated mass between 
60 and 200 thousand tons. The mass of the Tunguska meteorite (fell in Siberia in 1908) was at least 1 million tons, its speed $30-40 \mathrm{~km} / \mathrm{s}$; if we insert these values into Eq. (59), we obtain

$$
h_{\text {col }} \simeq 10^{-8} \mathrm{~m}
$$

corresponding values of $\delta g / g$ are still completely negligible (even in comparison with the variation of $g$ due to the Moon's tidal effect, Eq. (57)).

Let us now turn to another possible mechanism of the mirror matter motion. Can earthquakes cause translational oscillations of mirror matter around the Earth centre? Large enough earthquakes are known to excite free vibrations of the Earth; the study of these vibrations has become one of the most important pieces of information about the Earth interior (for more details see e.g. [9,14 [16]). These vibrations are classified into two categories:

- toroidal, in which only shear strain is present so that density is not perturbed; they are denoted by ${ }_{r} T_{l}$;

- spheroidal, where both shear and volume deformations arise, denoted by ${ }_{r} S_{l}$. The indexes $r$ and $l$ (for both $S$ and $T$ modes) have the same meaning as the radial and orbital quantum numbers of the hydrogen atom.

Toroidal modes do not lead to gravity perturbations so they cannot excite oscillations of mirror matter. On the other hand, spheroidal modes might cause the excitation of mirror matter oscillations through the gravitational coupling. Note that we should distinguish between two possible types of mirror matter oscillatons: a) bulk vibrations in which the centre of mass of mirror matter stays at rest in the Earth's centre and b) translational oscillations where the centre of mass of mirror matter oscillates around the Earth's centre in the Earth's gravitational field. We cannot say much about the spectrum of bulk vibrations without knowing the detailed structure of mirror matter (i.e., its density, elastic and dissipative properties etc.); for this reason we leave them out of our consideration. On the contrary, the period of translational oscillations can be found and is given by Eq.(50).

Our next task is to find out if there are any spheroidal Earth eigenmodes that could resonate with translational oscillations of mirror matter. Note that here we deal with the case of parametric resonance so we need to look for the eigenperiod $T_{E}=T_{1} / 2 \approx 1655 \mathrm{~s}$ rather than $T_{E}=T_{1}$ (the parametric resonance in the case $T_{E}=T_{1}$ is weaker than for $\left.T_{E}=T_{1} / 2\right)$. The closest such eigenmode is ${ }_{0} S_{4}$ with the period of [8]

$$
T\left({ }_{0} S_{4}\right)=1545.6 s
$$

In the time-varying gravitational field of ${ }_{0} S_{4}$ mode the frequency of translational mirror matter oscillations also becomes time dependent according to the law

$$
\omega^{2}(t)=\omega_{1}^{2}(1+a \cos \gamma t)
$$

where

$$
\omega_{1}=\frac{2 \pi}{T_{1}}, \quad \gamma=\frac{2 \pi}{T\left({ }_{0} S_{4}\right)}, \quad a=\frac{\delta \rho}{\rho},
$$

$\delta \rho / \rho$ is the amplitude of density variation in the ${ }_{0} S_{4}$ mode. 
The onset of parametric resonance is controlled by the quantity $s$ called amplification index (see e.g. [16]):

$$
s=\frac{1}{2} \sqrt{\left(\frac{a \omega_{1}}{2}\right)^{2}-\epsilon^{2}}, \quad \epsilon=\gamma-2 \omega_{1} .
$$

If the amplification index is real then the oscillation amplitude grows with time as exp st. In the opposite case $s^{2} \leq 0$ parametric resonance does not occur.

Using Eqs. (63,64, 65) we can find $s$ to be

$$
s=\frac{\omega_{1}}{4} \sqrt{\left(\frac{\delta \rho}{\rho}\right)^{2}-(0.28)^{2}},
$$

which is clearly imaginary. Thus we conclude that the condition for a parametric resonance is not satisfied and consequently there is no amplification of translational oscillations of the mirror matter.

It can be shown that the condition of parametric resonance with the ${ }_{0} S_{2}$-mode takes the following form:

$$
-\frac{5}{24} a^{2} \omega_{1}<\gamma^{\prime}-\omega_{1}<\frac{1}{24} a^{2} \omega_{1}
$$

where

$$
\gamma^{\prime}=\frac{2 \pi}{3233.25} s^{-1}
$$

is the frequency of the ${ }_{0} S_{2}$-mode. One can see that the condition (67) is not satisfied and there is no resonance with the ${ }_{0} S_{2}$-mode either.

\section{CONCLUSION}

We have investigated in detail geophysical constraints on the possible admixture of mirror matter inside the Earth. To this purpose, a method has been developed based on the Preliminary Reference Earth Model - the "Standard Model" of the Earth which describes its internal structure derived from the geophysical data in a systematic and self-consistent manner. If the density of the mirror matter is given, our method allows one to compute changes in various quantities characterising the Earth (such as its mass, moment of inertia, frequencies of its normal modes etc.). Comparing the computed and observed values of these characteristics, we can obtain for the first time the direct upper bounds on the possible concentration of the mirror matter in the Earth. In terms of the ratio of the mirror mass to the Earth mass these upper bounds range from $3.8 \times 10^{-4}$ to $3.8 \times 10^{-3}$ depending on the radius of the mirror matter ball. We then analyzed possible manifestations of mirror matter through the variations of the gravity acceleration on the Earth surface. These variations could arise as a result of an off-centre shift of the mirror matter due to several possible mechanisms such as lunar and solar tidal forces, meteorite impacts and earthquakes. Our estimates have shown that variations caused by these mechanisms are too small to be observed. 
In this work we have been based on a standard premise that mirror matter interacts with ordinary matter only gravitationally 円; we have not relied on any other specific assumptions about the mirror matter properties. Therefore our results are valid for other types of hypothetical matter coupled to ordinary matter by gravitation only; an example is shadow matter introduced in string theories. On the other hand, the use of equation of state and other macroscopic characteristic of mirror matter could lead to more severe constraints on the mirror mass inside the Earth.

\section{ACKNOWLEDGMENTS}

The authors are grateful to G.C.Joshi, R.Foot and B.H.J.McKellar for interesting discussions. This work was supported in part by the Australian Research Council.

\footnotetext{
${ }^{1}$ Note that mirror matter can also couple to ordinary matter through photon-mirror-photon mixing [4, 18]. An analysis of this interesting possibility is beyond the scope of the present work.
} 


\section{REFERENCES}

* e-mail: sasha@tauon.ph.unimelb.edu.au

$\dagger \quad$ e-mail: r.volkas@physics.unimelb.edu.au

[1] SuperKamiokande Collaboration, Y.Fukuda et al., Phys. Lett. B 433, 9 (1998); 436, 33 (1998); Phys. Rev. Lett. 81, 1562 (1998); 82, 2644 (1999).

[2] For a review see K.Zuber, Phys. Rep. 305, 295 (1998).

[3] For early works see T.D.Lee and C.N.Yang, Phys. Rev. 104, 254, (1956); I.Yu.Kobzarev, L.B.Okun and I.Ya.Pomeranchuk, Sov. J. Nucl. Phys. 3, 837 (1966); M.Pavsic, Int. J. Theor. Phys. 9, 229 (1974).

[4] R.Foot, H.Lew and R.R.Volkas, Phys. Lett. B 272, 67 (1991).

[5] R.Foot, H.Lew and R.R.Volkas, Mod. Phys. Lett. A 7, 2567 (1992); R.Foot, ibid. 9, 169 (1994); R.Foot and R.R.Volkas, Phys. Rev. D 52, 6595 (1995); see also Z.Silagadze, Phys. At. Nucl. 60, 272 (1997).

[6] For cosmological and astrophysical implications of mirror matter see: S.I.Blinnikov and M.Yu.Khlopov, Sov. J. Nucl. Phys. 36, 472 (1982); Sov. Astron. 27, 371 (1983); E.W.Kolb, D.Seckel and M.S.Turner, Nature (London) 314, 415, 1985; H.M.Hodges, Phys. Rev. D 47, 456 (1993); R.Foot and R.R.Volkas, Astropart. Phys. 7, 283 (1997); G.Matsas et al. hep-ph/9810456; N.F.Bell and R.R.Volkas, Phys. Rev. D 59, 107301 (1999); S.Blinnikov, astro-ph/9801015; astro-ph/9911138; R.Foot, Phys. Lett. B 452, 83 (1999); 471, 191 (1999) R.Foot and R.R.Volkas, Phys. Rev. D61, 043507 (2000)

[7] For models with broken mirror parity see R.Foot and H.Lew, preprint IP-ASTP-14 hepph/9411390 (unpublished); Z.Berezhiani and R.N.Mohapatra, Phys. Rev. D 52, 6607 (1995); Z.Berezhiani, A.D.Dolgov and R.N.Mohapatra, Phys. Lett. B 375, 26 (1996); Z.Berezhiani, Acta Phys. Polon. B 27, 1503 (1996); R.N.Mohapatra and V.Teplitz, Phys. Lett. B 462, 302 (1999); Ap. J. 478, 29 (1997); R.N.Mohapatra, S.Nussinov and V.L.Teplitz, astro-ph/9909376; see also: E.Akhmedov, Z.Berezhiani and G.Senjanovic, Phys. Rev. Lett. 69, 3013 (1992).

[8] A.M.Dziewonski and D.L.Anderson, Phys. Earth Planet. Interiors 25, 297, 1981.

[9] F.D.Stacey, Physics of the Earth, Brookfield Press, 1992 .

[10] D.L.Anderson, Theory of the Earth, Blackwell, 1989 .

[11] G.E.Backus and J.F.Gilbert, Geophys. J. R. Astr. Soc., 13, 247, 1967 .

[12] W.Torge, Gravimetry, de Gruyter, 1989 .

[13] D.Ya.Martynov, A Course of General Astrophysics, Nauka, 1971 (in Russian) .

[14] A.E.H.Love, A Treatise on the Mathematical Theory of Elasticity, Cambridge, 1927.

[15] V.I.Ferronsky, S.A.Denisik and S.V.Ferronsky, Jacobi Dynamics, D.Reidel, 1987.

[16] G.D.Garland, Introduction to Geophysics, Saunders, 1971.

[17] L.D.Landau and E.M.Lifshitz, Mechanics, Pergamon, 1986 .

[18] B.Holdom, Phys. Lett. B 166, 196 (1985); S.L.Glashow, Phys. Lett. B 167, 35 (1986); E.Carlson and S.L.Glashow, Phys. Lett. B 193, 168 (1987); M.Collie and R.Foot, Phys. Lett. B 432, 134 (1998). 